Article

\title{
Bacillus subtilis HU58 and Bacillus coagulans SC208 Probiotics Reduced the Effects of Antibiotic-Induced Gut Microbiome Dysbiosis in an M-SHIME ${ }^{\circledR}$ Model
}

\author{
Massimo Marzorati ${ }^{1,2}$, Pieter Van den Abbeele ${ }^{2}$, Sarah S. Bubeck ${ }^{3, *(D)}$, Thomas Bayne ${ }^{4}$, \\ Kiran Krishnan ${ }^{4}$, Aicacia Young ${ }^{4}$, Dilip Mehta ${ }^{5}$ and Anselm DeSouza ${ }^{5}$ \\ 1 Center for Microbial Ecology and Technology (CMET), Faculty of Bioscience Engineering, Ghent University, \\ Coupure Links 653, 9000 Ghent, Belgium; Massimo.Marzorati@prodigest.eu \\ 2 ProDigest, Technologiepark 82, 9052 Zwijnaarde, Belgium; Pieter.VandenAbbeele@prodigest.eu \\ 3 Bubeck Scientific, 194 Rainbow Drive \#9418, Livingston, TX 77399, USA \\ 4 Microbiome Labs, 101 E Town Pl, Saint Augustine, FL 92092, USA; tom@microbiomelabs.com (T.B.); \\ kiran@microbiomelabs.com (K.K.); ayoung@microbiomelabs.com (A.Y.) \\ 5 Synergia Life Sciences, PVT Ltd., Universal Majestic, 1503, PL Lokhande Marg, Chembur, Mumbai, \\ Maharashtra 400071, India; DilipMehta39@hotmail.com (D.M.); Kay2seven@gmail.com (A.D.) \\ * Correspondence: bubeckscientific@gmail.com
}

Received: 3 June 2020; Accepted: 7 July 2020; Published: 11 July 2020

\begin{abstract}
Benefits associated with probiotic use have been reported; however, the mechanisms behind these benefits are poorly understood. The effects of a probiotic formulation (MegaDuo ${ }^{\mathrm{TM}}$ ) containing Bacillus coagulans SC208 and Bacillus subtilis HU58 on intestinal permeability and immune markers was assessed using a combination of the in vitro gut model, the mucosal simulator of the human intestinal microbial ecosystem (M-SHIME ${ }^{\circledR}$ ), and an in vitro inflammatory bowel disease-like Caco-2/THP1 co-culture model in both healthy and antibiotic-induced dysbiosis conditions. Established M-SHIME ${ }^{\circledR}$ proximal colon vessels were treated with/without clindamycin (1 week) and then with/without daily MegaDuo ${ }^{\mathrm{TM}}$ treatment (2 weeks). The mucosal and luminal microbial communities were sampled weekly. Suspensions were removed from the proximal colon vessels after 1 and 2 weeks of MegaDuo ${ }^{\mathrm{TM}}$ treatment and added to the co-culture system. Transepithelial resistance (membrane barrier function), cytokine/chemokine release, and NFKB activity were then measured. Under conditions of antibiotic-induced dysbiosis, suspensions from MegaDuo ${ }^{\mathrm{TM}}$ treated vessels showed reduced gut membrane barrier damage and decreased levels of TNF $\alpha$ and IL-6 compared with suspensions from untreated vessels; no appreciable differences were observed under healthy conditions. MegaDuo ${ }^{\mathrm{TM}}$ treatment had no effect on NFkB activity of THP1-Blue ${ }^{\mathrm{TM}}$ cells. The potential benefits of MegaDuo ${ }^{\mathrm{TM}}$ treatment appeared most evident after 2 weeks of treatment.
\end{abstract}

Keywords: gut microbiome; gut barrier; gut inflammation; probiotics; immunomodulation; dysbiosis; M-SHIME

\section{Introduction}

The gut microbiome is a biologically active community that is important for maintaining intestinal homeostasis. Most of the organisms making up the gut microbiome reside in the colon [1] and they play important roles in nutrient and drug metabolism, immunomodulation, maintenance of gut barrier integrity, and protection from pathogenic bacteria [2]. A healthy gut microbiome should be highly diverse, stable, resistant to stress-related changes, and have a high level of redundancy for metabolic pathways [3,4]. 
The gut barrier is comprised of immune cells, intestinal bacteria, and epithelial cells held together by intercellular tight junctions. It protects against toxins and pathogenic bacterial and is involved in immunomodulation [2]. When intact, this barrier controls trafficking of molecules between the lumen of the gut and the lamina propria.

Short chain fatty acids (SCFAs), including acetate, propionate, and butyrate, are generated by bacterial fermentation in the colon where they exert beneficial effects on the intestinal epithelial barrier integrity and reduce intestinal inflammation [5]. In vitro, butyrate was shown to increase the transepithelial electrical resistance (TEER) of Caco-2 monolayers in a concentration-dependent manner [6]. Pro-inflammatory cytokines, like tumor necrosis factor (TNF) $\alpha$ and interleukin-1 $\beta$ (IL-1 $\beta$ ), significantly contribute to the inflammation observed in inflammatory bowel diseases (IBD) [7]. Furthermore, these pro-inflammatory cytokines induce the production of chemokines, like chemokine (C-X-C motif) ligand (CXCL)-10, IL-8, and monocyte chemoattractant protein 1 (MCP1), which are involved in neutrophil recruitment and reactive oxygen species production. Finally, a decrease in anti-inflammatory signaling also contributes to the development of IBD [8]. Indeed, IL-10 is a critical anti-inflammatory cytokine involved in maintaining intestinal immune homeostasis. IL-10 exerts pleiotropic immunosuppressive effects, as it inhibits the release of pro-inflammatory cytokines by macrophages, antigen presentation, and $\mathrm{T}$ cell differentiation. Furthermore, IL-10 promotes regulatory $\mathrm{T}$ cell differentiation. Intestinal inflammation has a significant impact on barrier integrity, and inflammation-induced barrier disruption can be mimicked in vitro by using a Caco-2/THP1 co-culture model [9]. In this model, anti-TNF $\alpha$ antibodies inhibited the THP1-induced barrier disruption, indicating the crucial role of TNF $\alpha$ in barrier dysfunction. Furthermore, butyrate decreased lipopolysaccharide (LPS)-induced TNF $\alpha$ and IL-1 $\beta$ production in THP1 cells, whereas it increased the LPS-induced IL-10 production and increased the LPS-induced NFKB activation in these cells [10]. Additionally, butyrate significantly increased toll-like receptor (TLR4)-mediated NFKB signaling in NCI-h716 cells by increasing the expression of the TLR4 receptor [11]. Finally, butyrate was shown to decrease pro-inflammatory cytokine and chemokine expression in several immune cell types [12].

Disruptions to a healthy gut microbiome can result in dysbiosis (gut microbiome imbalance), which is associated with many common diseases including IBD, irritable bowel syndrome, non-alcoholic fatty liver disease, and metabolic diseases such as obesity [13]. Exposure to antibiotics can cause dysbiosis of the gut microbiome, which results in a decrease in taxonomic diversity and changes in the numbers of individual microorganisms [14]. This imbalance can induce intestinal inflammatory responses, which may become pathological [15]. Antibiotics may also cause changes in the function of the gut barrier $[15,16]$. Such changes include translocation of gut bacteria across the colonic epithelium, which further contributes to increased inflammation [15].

Probiotics are defined as "live microorganisms which when administered in adequate amounts confer a health benefit to the host" [17]. They can help improve gastrointestinal health, improve gut barrier function, and affect both the mucosal and systemic immune systems [18]. Certain Bacillus strains have recently been recognized both as part of the normal flora and as having probiotic effects [19,20]. Bacillus spp. have several characteristics that make them suitable for probiotic use including a high level of resistance to desiccation and heat, as well as stability over a wide range of pHs [19]. Multiple health benefits have been attributed to the use of Bacillus spp. as probiotics, though the mechanisms of these benefits are poorly understood. Clinical trials evaluating the probiotic use of Bacillus spp. have been reviewed by Elshaghabee et al. [20].

Bacillus coagulans was first isolated from spoiled milk [21] and exhibits characteristics of both the Bacillus and the Lactobacillus genera [22]. It is a Gram-positive, rod shaped, spore-forming, facultative anaerobic, lactic-acid producing bacteria [22,23]. The probiotic activity observed for this species is likely due to lactic acid and other enzymes produced by the bacterium. B. coagulans has been reported to be safe by multiple regulatory agencies including the US Food and Drug Administration [24].

Bacillus subtilis has been isolated both from the soil and the intestinal tracts of humans [25-27]. It is a Gram-positive, rod shaped, spore-forming aerobic/facultative anaerobic bacterium [25]. B. coagulans 
and B. subtilis can be found either alone or in combination with other probiotics in multiple commercially available formulations [20]. It is thought that probiotics containing a combination of two or more spore-forming Bacillus species may improve the effects of treatment versus those containing a single strain. A recent study reported that when patients with dietary endotoxemia took a probiotic supplement containing five spore-forming species of Bacillus (Bacillus indicus HU36, B. subtilis HU58, B. coagulans, Bacillus licheniformis, and Bacillus clausii) for 30 days, they experienced a significant reduction in intestinal permeability as evidenced by significant reductions in endotoxins, triglycerides, and proinflammatory cytokines [28].

To support the use of probiotics in human health, it is important to understand specifically what benefits they impart. The aim of the present study was to assess the effects of MegaDuo ${ }^{\mathrm{TM}}$, a probiotic formulation containing B. coagulans SC208 and B. subtilis HU58, on intestinal permeability and immune markers using an in vitro gut model, the mucosal simulator of the human intestinal microbial ecosystem (M-SHIME $\left.{ }^{\circledR}\right)$ [29], in combination with an in vitro IBD-like Caco-2/THP1 co-culture model [9] under healthy gut conditions or following antibiotic-induced dysbiosis.

\section{Materials and Methods}

\subsection{M-SHIME ${ }^{\circledR}$ Model.}

The M-SHIME ${ }^{\circledR}$ reactor configuration was adapted from the SHIME ${ }^{\circledR}$ model (ProDigest, Ghent, Belgium and Ghent University, Ghent, Belgium) previously described by Molly et al. [30] to include modelling of both the mucus-associated and luminal microbial communities [29,31]. Details of inoculum preparation, retention times, $\mathrm{pH}$, temperature settings, mucin-covered microcosms, and reactor feed composition have been previously described [29,32]. The present study included minor modifications to Van den Abbeele et al. [29], which are described as follows. Briefly, the reactor consisted of three double-jacketed vessels that were connected through peristaltic pumps and operated completely anaerobically. These vessels simulate the stomach and small intestine, and for the purposes of our study, the colon vessels were limited to the proximal colon ( $\mathrm{pH} 5.6-5.9$, retention time $20 \mathrm{~h}$ ). To simulate the gut microbiome, the proximal colon vessels were inoculated with microbiota isolated from an appropriate fecal sample originating from a single healthy adult volunteer with no history of antibiotic treatment in the 6 months prior to sample collection.

The first vessel was fed nutritional medium supplemented with $2 \mathrm{~g} / \mathrm{L}$ L-alanine to allow optimal germination of Bacillus spores. During the simulation of the small intestine, the system was fed pancreatic and bile liquid. Feedings were conducted three times a day using a fill-and-draw method. After digestion in the upper gastrointestinal tract, the slurry was pumped into the proximal colon vessel. Proximal colon vessels were continuously stirred and monitored for constant volume and $\mathrm{pH}$. All vessels were maintained at $37^{\circ} \mathrm{C}$.

The experimental schedule is schematically shown in Figure 1. There was a 2-week stabilization period to allow the microbiota to adapt to the in vitro environment, followed by a 2-week control period during which stability in the microbiome was established and baseline parameters were measured. At the completion of the control period, there was a 1-week pre-treatment period. Clindamycin (33.9 $\mathrm{mg} / \mathrm{mL}$, thrice daily) was added to one proximal colon vessel for 7 days to induce dysbiosis of the microbiota, which is characteristic of many gastrointestinal tract diseases. The other proximal colon was operated under normal healthy conditions, without antibiotics. Next, the 2-week treatment period was initiated, and MegaDuo ${ }^{\mathrm{TM}}$ (combination of B. coagulans SC208 and B. subtilis HU58; powder dissolved in gastric fluid; Synergia Life Sciences, Mumbai, Maharashtra, India) was administered once daily (first feeding cycle, $2 \times 10^{9} \mathrm{CFU} /$ day) over the 2-week treatment period. Proximal colon samples were collected after 1 and 2 weeks of treatment. Supernatants were centrifuged ( 5 min, 9000 RPM) and stored at $-20{ }^{\circ} \mathrm{C}$. 


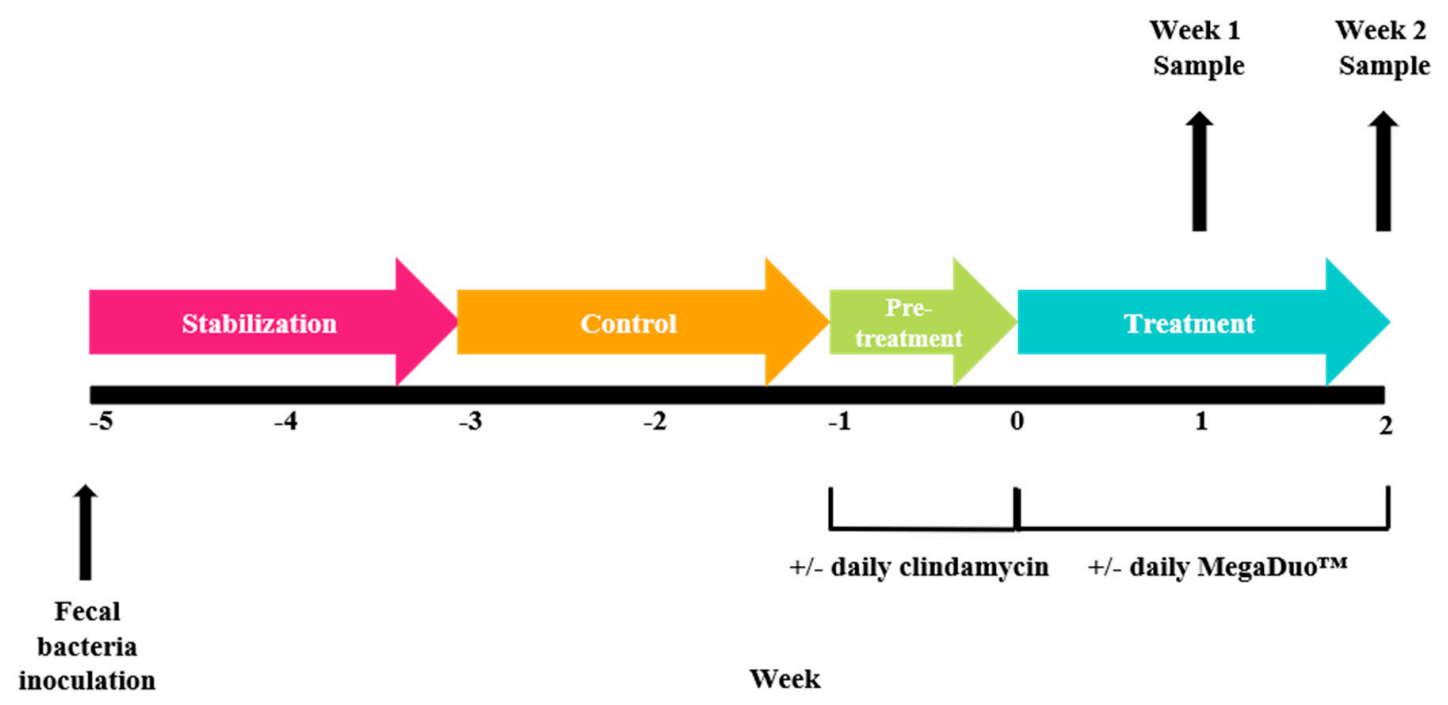

Figure 1. Schematic of the experimental design.

\subsection{Microbial Community Analysis}

Once per week, from the start of the control period to the end of the treatment period, samples were collected for microbial community analysis from both the luminal and mucosal compartment of each vessel. DNA was isolated from $0.1 \mathrm{~g}$ of the mucosal compartment sample or pelleted cells originating from a $1 \mathrm{~mL}$ luminal sample using the method described by Vilchez-Vargas et al. [33]. Next, quantitative polymerase chain reaction (qPCR) for Bacteroides phylum, Firmicutes phylum, Enterobacteriaceae family, Bifidobacterium spp., and Lactobacillus spp. was performed using a StepOnePlus ${ }^{\mathrm{TM}}$ real-time PCR system (Applied Biosystems, Foster City, CA, USA). Each sample was analyzed in technical triplicate, and outliers (more than $1 \mathrm{CT}$ difference) were omitted. qPCR was conducted according to previously described methods: Firmicutes and Bacteroidetes phyla, Guo et al. [34]; Bifidobacterium spp., Rintillä et al. [35]; Lactobacillus spp., Furet et al. [36]; Enterobacteriaceae, Nakano et al. [37].

Microbiota profiling of the colon compartments was accomplished using 16S-targeted Illumina sequencing analysis. The $16 \mathrm{~S}$ rRNA gene V3-V4 hypervariable regions were amplified by PCR using primers 341F (5'-CCT ACG GGN GGC WGC AG-3') and 785Rmod (5'-GAC TAC HVG GGT ATC TAA KCC-3'); to increase coverage, the reverse primer was adapted from Klindworth et al. [38]. Quality control PCR was performed using Taq DNA polymerase with the Fermentas PCR Kit according to the manufacturer's instructions (Thermo Fisher Scientific, Waltham, MA, USA). Genomic DNA extracts $(10 \mu \mathrm{L})$ were sent to LGC genomics $\mathrm{GmbH}$ (Germany) for library preparation and sequencing on an Illumina MiSeq platform with v3 chemistry with the above-mentioned primers [38].

Read assembly and cleanup for the 16S-targeted sequencing analysis was derived from the MiSeq protocol described by the Schloss lab [39,40]. In brief, mothur (v. 1.39.5) was used to assemble reads into contigs, perform alignment-based quality filtering (alignment to the mothur-reconstructed SILVA SEED alignment, v. 123), remove chimeras, assign taxonomy using a naive Bayesian classifier [41] and SILVA NR v128, and cluster contigs into operational taxonomic units (OTUs) at 97\% sequence similarity. All sequences classified as Eukaryota, Archaea, chloroplasts, and mitochondria were removed, as well as sequences that could not be classified. For each OTU, representative sequences were selected as the most abundant sequence within that OTU.

\subsection{Caco-2/THP1-Blue ${ }^{\mathrm{TM}}$ Co-Culture Model.}

Co-culture experiments using Caco-2 (HTB-37, American Type Culture Collection, Manassas, VA, USA) and THP1-Blue ${ }^{\mathrm{TM}}$ cells (InvivoGen, San Diego, CA, USA) were performed as previously described [42]. Briefly, a semi-permeable insert (pore size $0.4 \mu \mathrm{M}$ ) with a monolayer of Caco-2 cells (TEER $>300 \Omega \mathrm{cm}^{2}$ (epithelial volt-ohm meter, Millipore, Burlington, MA, USA)) was placed into a 
well of phorbol 12-myristate 13-acetic acid (PMA)-differentiated THP1-Blue ${ }^{\mathrm{TM}}$ cells (100 ng/mL, $\left.48 \mathrm{~h}\right)$. The apical compartment (containing Caco-2 cells) was filled with sterile-filtered $(0.22 \mu \mathrm{M})$ colonic M-SHIME ${ }^{\circledR}$ suspensions (diluted 1:5 v/v in Caco-2 complete medium) or treated with $12 \mathrm{mM}$ sodium butyrate $(\mathrm{NaB})$ (Sigma-Aldrich, St. Louis, MO, USA) for a positive control and complete Caco-2 medium for a negative control. The basolateral compartment (containing THP1-Blue ${ }^{\mathrm{TM}}$ cells) was filled with complete Caco-2 medium. Co-cultures were then placed in a humidified incubator at $37^{\circ} \mathrm{C}$, $5 \% \mathrm{CO}^{2}$. Twenty-four hours later, the TEER was measured for each well. The percent of the initial value $(0 \mathrm{~h})$ for each well was calculated using the following formula:

$$
\left(24 \mathrm{~h} \Omega \mathrm{cm}^{2}-\text { empty insert } \Omega \mathrm{cm}^{2} / 0 \mathrm{~h} \Omega \mathrm{cm}^{2}\right) \times 100
$$

The basolateral media was then discarded and replaced with Caco-2 complete medium containing $500 \mathrm{ng} / \mathrm{mL}$ ultrapure LPS (Escherichia coli K12, InvivoGen, San Diego, CA, USA) to stimulate the cells. Medium containing $500 \mathrm{ng} / \mathrm{mL}$ LPS and $1 \mu \mathrm{M}$ hydrocortisone (HC, Sigma-Aldrich, St. Louis, MO, USA) or medium without LPS were used as controls. After $6 \mathrm{~h}$, the basolateral supernatants were collected and processed for the measurement of cytokines, chemokines, and NFkB. Co-culture experiments were performed in technical triplicates

\subsection{Measurement of NFKB Activity}

THP1-Blue ${ }^{\mathrm{TM}}$ cells are stably transfected with an $\mathrm{NF}_{k} \mathrm{~B}$ reporter construct in which the secreted alkaline phosphatase (SEAP) gene is under the control of an NFkB inducible promoter. When NFKB is activated, expression of SEAP is induced. SEAP was measured in co-culture basolateral supernatants using the QUANTI-Blue reagent (InvivoGen, San Diego, CA, USA) according to the manufacturer's instructions.

\subsection{Cytokine and Chemokine Measurements}

Levels of human IL-1 $\beta$, IL-8, IL-10, TNF $\alpha$, CXCL10, and MCP1 in the co-culture basolateral supernatants were measured using Luminex ${ }^{\circledR}$ multiplex (Affymetrix-eBioscience, Waltham, MA, USA). Assays were performed according to the manufacturer's instructions.

\subsection{Statistical Methods}

For the TEER controls, complete Caco-2 media (negative) and $\mathrm{NaB}$ (positive) values were compared using an unpaired, two-tailed Student's $t$-test. For the co-cultures treated with colonic M-SHIME ${ }^{\circledR}$ suspensions, treatment samples were compared to the M-SHIME ${ }^{\circledR}$ control (M-SHIME ${ }^{\circledR}$ media) using an ordinary one-way analysis of variance (ANOVA) with Dunnet's multiple comparisons test. Regarding the controls, cytokine levels and NFKB activity, LPS-, LPS + HC, and LPS + NaB were compared to LPS+; for the colonic M-SHIME ${ }^{\circledR}$ suspensions, treatment samples were compared to the M-SHIME ${ }^{\circledR}$ control (M-SHIME ${ }^{\circledR}$ media) using an ordinary one-way ANOVA with Dunnett's multiple comparisons test. A $p$-value $<0.05$ was considered statistically significant; ${ }^{*}{ }^{* *},{ }^{* * *}$, and ${ }^{* * * *}$ represent $p<0.05$, $p<0.01, p<0.001$ and $p<0.0001$, respectively. All statistics were performed using GraphPad Prism version 7.02 for Windows (GraphPad Software, San Diego, CA, USA).

\section{Results}

\subsection{Microbial Community Analysis}

The luminal microbiota composition for the duration of the study is shown in Figure 2. Lactobacillus and Bifidobacterium spp. remained constant throughout the study period under healthy conditions (no clindamycin). The addition of clindamycin decreased these levels, and they did not recover after cessation of antibiotic treatment, even with MegaDuo ${ }^{\mathrm{TM}}$ treatment. Under healthy conditions, Bacteroidetes and Firmicutes levels were increased upon treatment with MegaDuo ${ }^{\mathrm{TM}}$. No clear 
effects on the Bacteroidetes levels, and a decrease in Firmicutes levels occurred with clindamycin exposure. Firmicutes levels recovered in both the MegaDuo $^{\mathrm{TM}}$ treated and M-SHIME ${ }^{\circledR}$ control lumens. Under healthy conditions, Enterobacteriaceae levels gradually increased in both the MegaDuo ${ }^{\mathrm{TM}}$ treated and M-SHIME ${ }^{\circledR}$ control lumens, and clindamycin exposure resulted in an increase in these levels. After cessation of clindamycin, MegaDuo ${ }^{\mathrm{TM}}$ treatment resulted in an immediate decrease of Enterobacteriaceae, indicating a faster recovery of these levels compared with the M-SHIME ${ }^{\circledR}$ control. This is of importance as this family is most likely linked with enteric diseases.

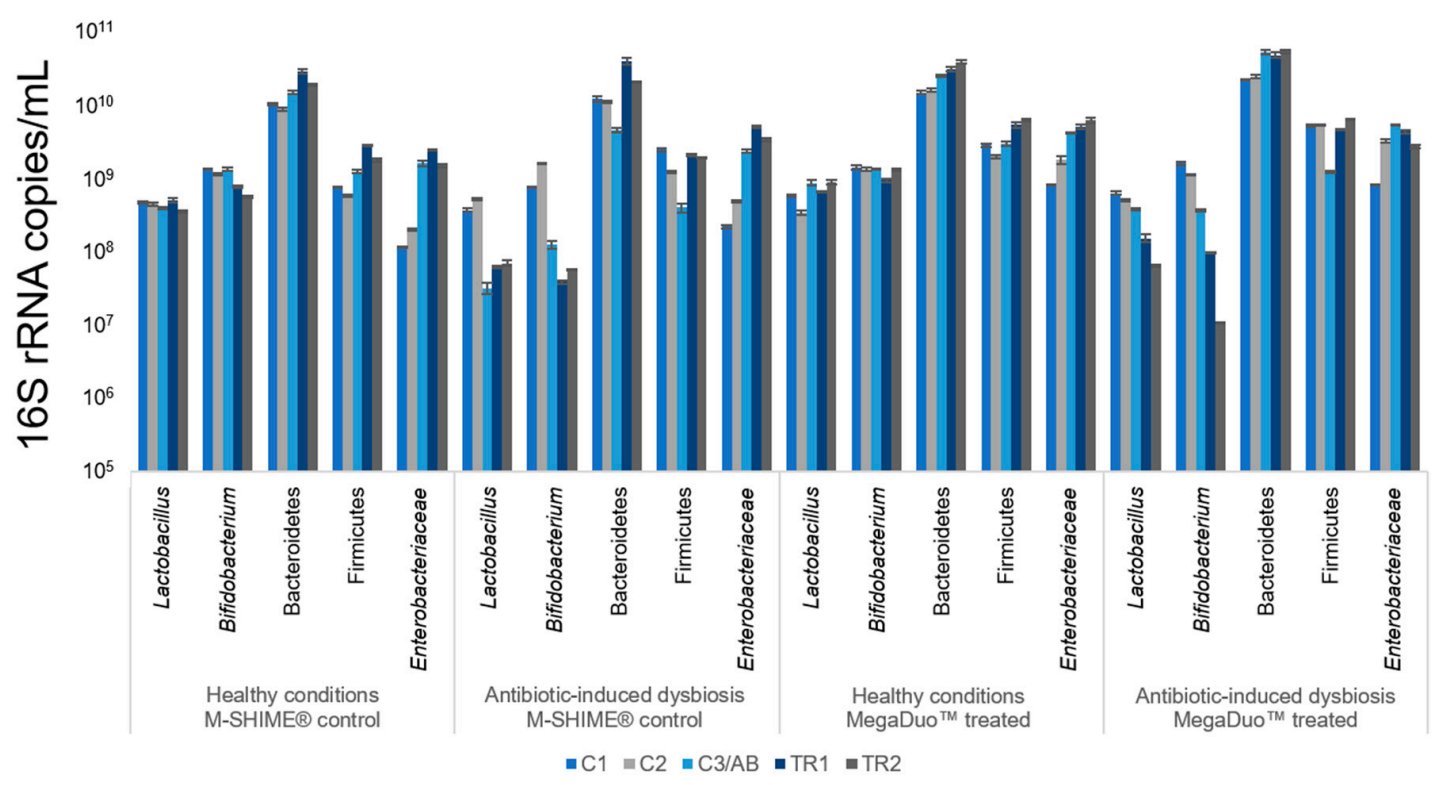

Figure 2. Luminal microbial community composition as assessed by qPCR. All data are shown as $16 \mathrm{~S}$ rRNA copies/mL in the luminal phase of the proximal colon. All samples were analyzed in triplicate. Data are shown as mean \pm standard deviation. $\mathrm{C} 1=$ control Week $1 ; \mathrm{C} 2=$ control Week 2; $\mathrm{C} 3 / \mathrm{AB}=$ control Week 3 (healthy condition, pre-treatment)/antibiotic (antibiotic-induced dysbiosis condition, pre-treatment); TR1 = treatment Week 1; TR2 = treatment Week 2; M-SHIME ${ }^{\circledR}=$ mucosal simulator of the human intestinal microbial ecosystem.

The mucosal microbiota composition for the duration of the study is shown in Figure 3. Under healthy conditions, there were no notable differences in microbiota composition between MegaDuo $^{\mathrm{TM}}$ treated and M-SHIME ${ }^{\circledR}$ control chambers. After clindamycin exposure, most bacterial groups increased, indicating a protective effect of the mucosal environment in terms of antibiotic exposure. After cessation of antibiotic treatment, similar effects as reported for the luminal microbiota were observed with MegaDuo ${ }^{\mathrm{TM}}$ treatment.

Microbial community composition at the family and OTU level is shown in Figure 4. Under healthy conditions, MegaDuo ${ }^{\mathrm{TM}}$ treatment resulted in a decrease in abundance of the Bifidobacteriaceae and the Veillonellaceae families and an increase in abundance of the Prevotellaceae and Baccillaceae families in both the luminal and mucosal environment. There was also an increase in the abundance of Lactobacillaceae in the mucus layer. Clindamycin exposure resulted in a decrease in Actinobacteria abundance and an increase in Enterobacteriaceae abundance; Prevotellaceae levels decreased below the detection limit in both the luminal and mucosal environments. There was also a drastic negative effect on the Firmicutes phylum. Treatment with MegaDuo ${ }^{\mathrm{TM}}$ under dysbiosed conditions resulted in an increase in bacterial families associated with propionate (SCFA) production, while Enterobacteriaceae levels decreased. There was an increase in the abundance of the Bacillaceae family in the lumen. Reciprocal Simpson Diversity Index data indicated that antibiotic treatment reduced the diversity of the gut microbial community, with the strongest effect in the mucosal compartment; $\mathrm{MegaDuo}^{\mathrm{TM}}$ increased this diversity, particularly following antibiotic treatment (Supplementary Materials Table S1). 


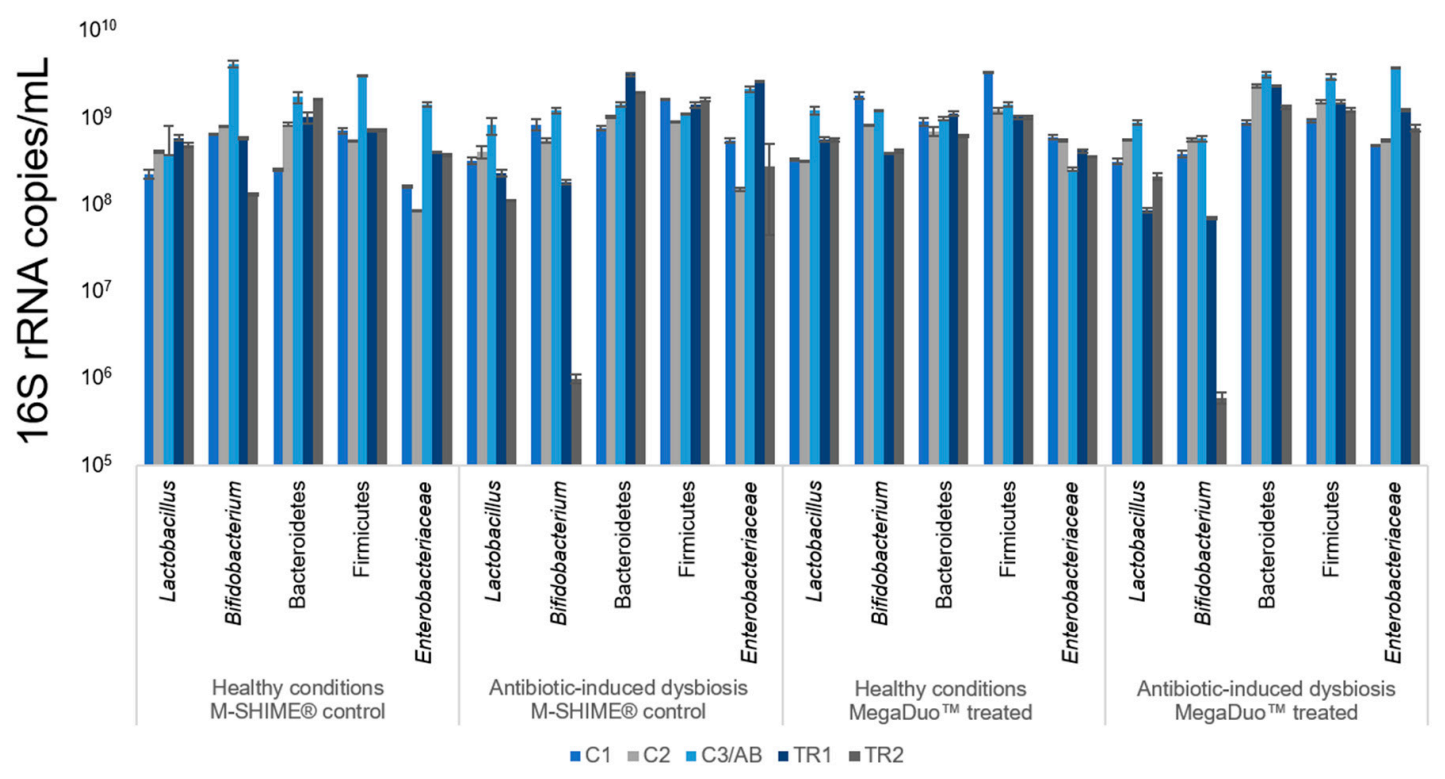

Figure 3. Mucosal microbial community composition as assessed by qPCR. All data are shown as $16 \mathrm{~S}$ rRNA copies/mL in the mucosal phase of the proximal colon. All samples were analyzed in triplicate. Data are shown as mean \pm standard deviation. $\mathrm{C} 1=$ control Week $1 ; \mathrm{C} 2=$ control Week 2; $\mathrm{C} 3 / \mathrm{AB}=$ control Week 3 (healthy condition, pre-treatment)/antibiotic (antibiotic-induced dysbiosis condition, pre-treatment); TR1 = treatment Week 1; TR2 = treatment Week 2; M-SHIME ${ }^{\circledR}=$ mucosal simulator of the human intestinal microbial ecosystem.

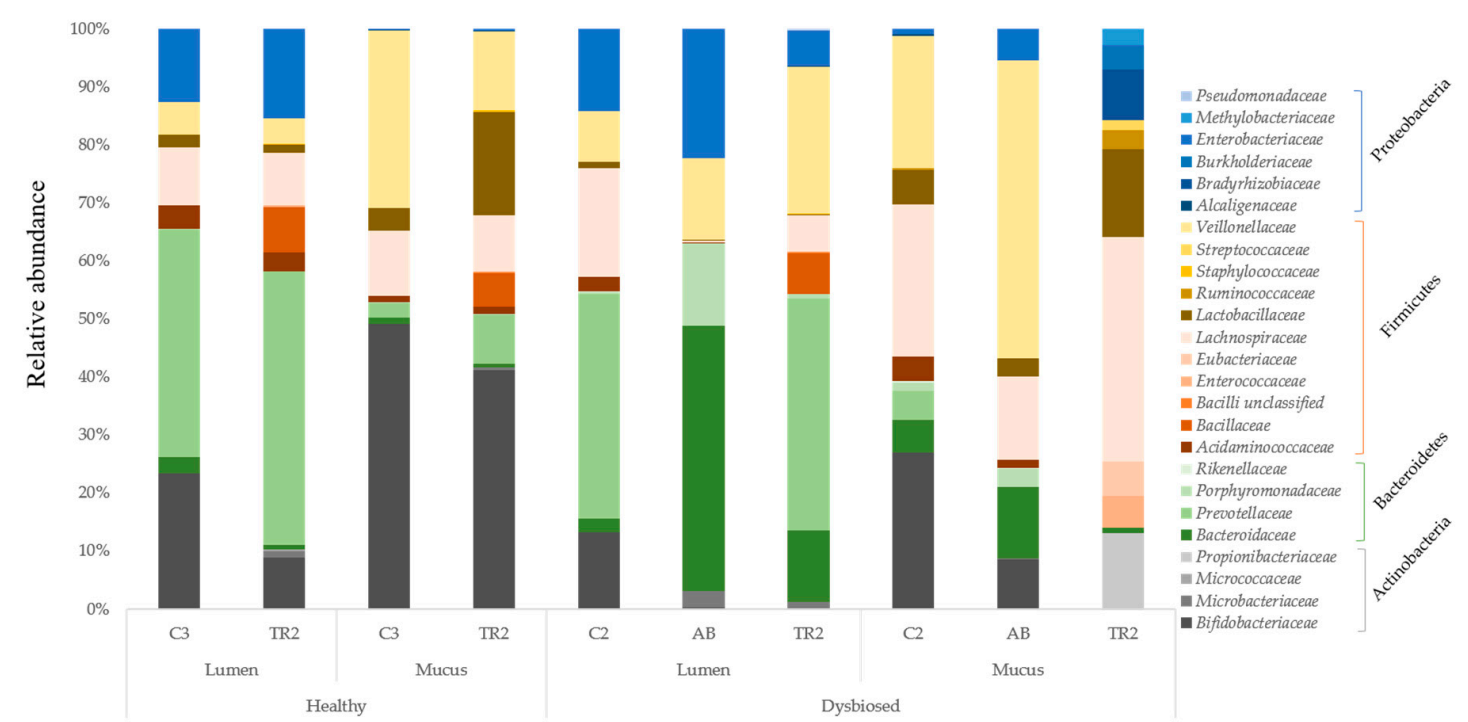

Figure 4. Relative abundance of different families belonging to specific phyla in the lumen and mucus of the proximal colon of the M-SHIME ${ }^{\circledR}$ treated with MegaDuo ${ }^{\mathrm{TM}}$ under healthy and antibiotic-induced dysbiosed conditions. $\mathrm{C} 3$ = end of the control period (healthy condition); TR2 = end of the treatment period; $\mathrm{C} 2$ = end of the control period (dysbiosed condition); $\mathrm{AB}=$ end of the antibiotic period; M-SHIME ${ }^{\circledR}=$ mucosal simulator of the human intestinal microbial ecosystem.

\subsection{Transepithelial Electrical Resistance}

Caco-2/THP1-Blue ${ }^{\mathrm{TM}}$ co-cultures were exposed to controls or M-SHIME ${ }^{\circledR}$ suspensions (+/- clindamycin pretreatment, 1 or 2 weeks of daily $+/-$ MegaDuo $^{\mathrm{TM}}$ dosing) for $24 \mathrm{~h}$, and then THP1-induced damage to the Caco-2 membrane was measured. The controls (complete Caco-2 medium and $\mathrm{NaB}$ ) performed as expected (Supplementary Materials Figure S1). NaB treatment protected the Caco-2 cells from THP1-induced damage, maintaining the barrier integrity of the monolayer; the TEER 
was significantly higher in the $\mathrm{NaB}$ treated co-cultures compared with the Caco-2 medium control $(p<0.0001)$.

Exposure of the co-cultures to +clindamycin suspensions without MegaDuo ${ }^{\mathrm{TM}}$ dosing resulted in THP1-induced damage to the Caco-2 membrane barrier at a level similar to that observed for the complete Caco-2 media control, while exposure to +clindamycin suspensions dosed with MegaDuo ${ }^{\mathrm{TM}}$ resulted in protection of the Caco- 2 membrane integrity relative to both the M-SHIME ${ }^{\circledR}$ and Caco- 2 media controls (Figure 5a). The protection was significant whether the suspensions were collected 1 week $(p=0.0314)$ or 2 weeks $(p=0.0002)$ after MegaDuo ${ }^{\mathrm{TM}}$ dosing $\left(\right.$ MegaDuo $^{\mathrm{TM}}$ vs. M-SHIME $^{\circledR}$ control). The effect was more pronounced at 2 weeks, with MegaDuo ${ }^{\mathrm{TM}}$ exposed co-cultures showing a Caco-2 membrane TEER value $70 \%$ of the initial value (pre-exposure). The controls (M-SHIME ${ }^{\circledR}$ control or Caco-2 media) had TEER values closer to $60 \%$. The results demonstrated a protective effect of MegaDuo $^{\mathrm{TM}}$ treated M-SHIME ${ }^{\circledR}$ suspensions against THP1-induced damage to the Caco-2 membrane barrier under conditions of antibiotic-induced dysbiosis.

M-SHIME ${ }^{\circledR}$ suspensions that were not pretreated with clindamycin, whether or not they were dosed with MegaDuo ${ }^{\mathrm{TM}}$, were largely able to prevent THP1-induced damage to the membrane barrier integrity. Co-cultures exposed to the M-SHIME ${ }^{\circledR}$ suspensions had TEER values of $80-90 \%$ of pre-exposure values compared with $57 \%$ for the co-cultures treated with complete Caco-2 media (Figure 5b). This indicates that in conditions representing a healthy microbiome, the metabolites present in the M-SHIME ${ }^{\circledR}$ suspensions were sufficient to protect membrane barrier integrity. Note that the control M-SHIME ${ }^{\circledR}$ supernatants at Week 1 were suspected to have been contaminated during sample processing (i.e., after the collection of the samples) and were therefore not included in the analysis. The microbiota composition was similar for the control M-SHIME ${ }^{\circledR}$ vessel at Week 1 and Week 2 (Figures 2 and 3, TR1 vs. TR2), allowing for the use of Week 2 data as a comparator in place of Week 1 data.

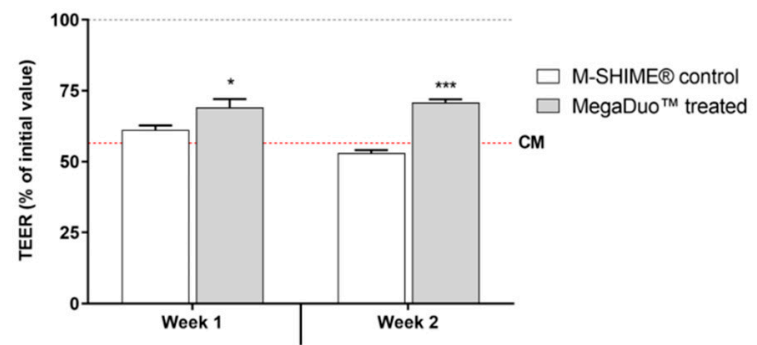

(a)

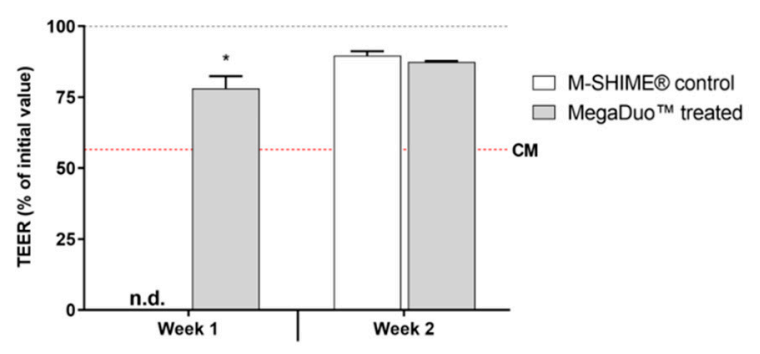

(b)

Figure 5. Barrier integrity of Caco-2 cells after exposure to M-SHIME ${ }^{\circledR}$ suspensions (a) with or (b) without pre-treatment with clindamycin. ${ }^{*} p \leq 0.05,{ }^{* * *} p \leq 0.001$, grey dashed line $=100 \%$ TEER, red dashed line $=$ TEER $(\%$ of initial value) for co-cultures treated with CM. Error bars represent standard error of the mean. $\mathrm{CM}=\mathrm{Caco}-2$ media; $\mathrm{M}-\mathrm{SHIME}{ }^{\circledR}=$ mucosal simulator of the human intestinal microbial ecosystem; TEER $=$ transepithelial electric resistance.

\subsection{Immune Markers}

Caco-2/THP1-Blue ${ }^{\mathrm{TM}}$ co-cultures were exposed to controls or M-SHIME ${ }^{\circledR}$ suspensions (+/- clindamycin pretreatment, after 1 or 2 weeks of daily $+/-$ MegaDuo $^{\mathrm{TM}}$ dosing) for $24 \mathrm{~h}$, and then LPS was added to the cultures; $6 \mathrm{~h}$ later, supernatants were collected and assayed for cytokines and chemokines. The controls worked as expected. LPS significantly increased secretion of all cytokines and chemokines tested relative to the complete Caco-2 media control except IL-10, which was significantly reduced (LPS+ vs. LPS-: TNF $\alpha, p=0.003$; IL-8, $p=0.0194 ; \mathrm{MCP} 1, p=0.0245 ;$ CXCL10, $p=0.0005$; IL-10, $p=0.0054$; IL-6, $p<0.0001$ ). HC was able to significantly suppress LPS-induced cytokines and chemokines (LPS+ vs. LPS+HC: TNF $\alpha, p=0.004 ;$ IL-8, $p=0.0069 ; \mathrm{MCP} 1, p=0.0078$; CXCL10, $p=0.0017$; IL-10, $p=0.0076$; IL-6, $p<0.0001)$. NaB had selective effects; it was able to significantly increase LPS-induced IL-10 $(p<0.0001)$ and IL-6 $(p=0.0124)$ and to significantly decrease LPS-induced 
$\operatorname{TNF} \alpha(p=0.003), \operatorname{IL}-8(p=0.0421), \operatorname{MCP1}(p=0.0011)$ and CXCL10 $(p=0.0004)$, which are involved in inflammation and recruitment of immune cells (Supplementary Materials Figures S2A-F).

The effects of M-SHIME ${ }^{\circledR}$ suspensions on cytokine and chemokine release in the co-culture system are reported in Tables 1 and 2. The levels of some LPS-induced cytokines and chemokines were differentially affected by MegaDuo ${ }^{\mathrm{TM}}$ and control M-SHIME ${ }^{\circledR}$ suspensions that were pretreated with clindamycin. We observed no differences in the levels of TNF $\alpha$, IL-8, or IL-10, a slight reduction in MCP1 and CXCL10, and a significant reduction in IL-6 $(p=0.007)$ for MegaDuo ${ }^{\mathrm{TM}}$ treated versus M-SHIME ${ }^{\circledR}$ control suspensions collected after 1 week of daily MegaDuo ${ }^{\mathrm{TM}}$ dosing. After 2 weeks of daily dosing, we observed no differences in IL-8, MCP1, CXCL10, or IL-6, a slight increase in IL-10, and a significant reduction in TNF $\alpha(p=0.0067)$ for MegaDuo $^{\mathrm{TM}}$ treated versus M-SHIME ${ }^{\circledR}$ control suspensions (Table 1).

Table 1. Effect of M-SHIME ${ }^{\circledR}$ supernatants on cytokine and chemokine release in the co-culture system under conditions of dysbiosis.

\begin{tabular}{|c|c|c|c|c|c|c|}
\hline \multirow{3}{*}{$\begin{array}{l}\text { Cytokine/ } \\
\text { Chemokine }\end{array}$} & \multicolumn{6}{|c|}{ Dysbiosis (Clindamycin Pre-treatment) } \\
\hline & \multicolumn{3}{|c|}{ Week 1} & \multicolumn{3}{|c|}{ Week 2} \\
\hline & $\begin{array}{c}\text { M-SHIME } \\
\text { Control }\end{array}$ & $\begin{array}{l}\text { MegaDuo } \\
\text { Treated }\end{array}$ & $p$ Value & $\begin{array}{c}\text { M-SHIME } \\
\text { Control }\end{array}$ & $\begin{array}{l}\text { MegaDuo } \\
\text { Treated }\end{array}$ & $p$ Value \\
\hline $\mathrm{TNF} \alpha^{\mathrm{a}}$ & 6585.5 (194.7) & $6970.0(305.4)$ & 0.8227 & $9172.6(622.3)$ & $6604.7(343.9)$ & 0.0067 \\
\hline IL-8 ${ }^{\mathrm{b}}$ & $336.2(50.2)$ & $343.7(51.2)$ & 0.9906 & $372.3(21.5)$ & $391.9(29.6)$ & 0.9502 \\
\hline $\mathrm{MCP}^{\mathrm{a}}$ & $7068.4(1129.6)$ & $4071.1(696.0)$ & 0.0699 & $8388.8(601.0)$ & $8032.9(835.3)$ & 0.9480 \\
\hline CXCL10 ${ }^{a}$ & $781.5(78.6)$ & $641.5(124.3)$ & 0.4049 & $563.8(29.4)$ & $555.21(23.5)$ & 0.9962 \\
\hline IL-10 a & $10.3(0.4)$ & $10.7(0.9)$ & 0.8938 & $9.9(0.8)$ & $12.1(0.4)$ & 0.0777 \\
\hline IL-6 ${ }^{\mathrm{a}}$ & $510.6(14.7)$ & $370.3(26.4)$ & 0.0070 & $483.5(24.0)$ & $483.9(29.5)$ & $>0.999$ \\
\hline
\end{tabular}

All values are given as mean (standard error of the mean). ${ }^{a}$ Values are in $\mathrm{pg} / \mathrm{mL} .{ }^{\mathrm{b}}$ Values are in $\mathrm{ng} / \mathrm{mL}$. M-SHIME ${ }^{\circledR}=$ mucosal simulator of the human intestinal microbial ecosystem.

In the absence of clindamycin pretreatment (representing healthy gut conditions), exposure of co-cultures to suspensions after MegaDuo ${ }^{\mathrm{TM}}$ treatment resulted in no change for CXCL10, IL-10, or IL-6 and a significant reduction in TNF $\alpha(p=0.0047)$, IL-8 $(p=0.0238)$, and MCP1 $(p=0.0359)$ in M-SHIME ${ }^{\circledR}$ suspensions after 1 week of treatment (compared with M-SHIME ${ }^{\circledR}$ control suspensions collected at Week 2; data collected using M-SHIME ${ }^{\circledR}$ control suspensions from Week 1 were suspected to have been contaminated during sample processing (i.e., after sample collection) and were therefore not used for data analysis). The microbiota composition was similar for the control M-SHIME vessel at Week 1 and Week 2 (Figures 2 and 3, TR1 vs. TR2), allowing for the use of Week 2 data as a comparator in place of Week 1 data. There was no change in the levels of TNF $\alpha$, IL-8, CXCL10, IL-10, and IL-6 and a significant increase in MCP1 $(p=0.0304)$ after 2 weeks of treatment compared with the M-SHIME ${ }^{\circledR}$ control (Table 2).

The supernatants collected for the cytokine and chemokine assays were used to determine the NFKB activity of THP1-Blue ${ }^{\mathrm{TM}}$ cells. The controls worked as expected (Supplementary Materials Figure S3). LPS increased NFKB activity (LPS+ vs. LPS-; $p=0.0020$ ), and HC was able to reduce LPS-induced activity (LPS+ vs. LPS $+\mathrm{HC} ; p=0.0271$ ). NaB increased NFkB activity in the presence of LPS (LPS+ vs. LPS $+\mathrm{NaB} ; p=0.0003$ ). 
Table 2. Effect of M-SHIME ${ }^{\circledR}$ supernatants on cytokine and chemokine release in the co-culture system under healthy conditions.

\begin{tabular}{|c|c|c|c|c|c|c|}
\hline \multirow{3}{*}{$\begin{array}{l}\text { Cytokine/ } \\
\text { Chemokine }\end{array}$} & \multicolumn{6}{|c|}{ Healthy } \\
\hline & \multicolumn{3}{|c|}{ Week 1} & \multicolumn{3}{|c|}{ Week 2} \\
\hline & $\begin{array}{c}\text { M-SHIME }{ }^{\circledR} \\
\text { Control }\end{array}$ & $\begin{array}{l}\text { MegaDuo } \\
\text { Treated }\end{array}$ & $p$ Value $^{1}$ & $\begin{array}{l}\text { M-SHIME } \\
\text { Control }\end{array}$ & $\begin{array}{l}\text { MegaDuo } \\
\text { Treated }\end{array}$ & $p$ Value \\
\hline $\mathrm{TNF} \alpha^{2}$ & n.d. & $5827.8(202.2)$ & 0.0047 & $7251.4(275.6)$ & 7124.6 (137.4) & 0.9140 \\
\hline IL- $8^{3}$ & n.d. & $249.6(61.6)$ & 0.0238 & $427.3(22.0)$ & $372.9(35.8)$ & 0.5785 \\
\hline $\mathrm{MCP}^{2}{ }^{2}$ & n.d. & $5778.6(331.5)$ & 0.0359 & 8595.7 (339.3) & $11,516.0(1212.1)$ & 0.0304 \\
\hline $\mathrm{CXCL10}^{2}$ & n.d. & $645.3(23.8)$ & 0.9326 & $655.9(22.5)$ & $703.0(18.5)$ & 0.3057 \\
\hline IL-10 2 & n.d. & $14.9(1.3)$ & 0.3786 & $17.0(1.0)$ & $19.8(1.0)$ & 0.2049 \\
\hline IL-6 ${ }^{2}$ & n.d. & $746.4(34.4)$ & 0.9165 & 764.7 (31.9) & $865.7(36.5)$ & 0.1294 \\
\hline
\end{tabular}

All values are given as mean (standard error of the mean). ${ }^{1}$ Week $1 \mathrm{MegaDuo}^{\mathrm{TM}}$ treated vs. Week $2 \mathrm{M}-\mathrm{SHIME}{ }^{\circledR}$ control; Week 1 M-SHIME ${ }^{\circledR}$ control suspensions were suspected to have been contaminated during sample processing (i.e., after sample collection) so the data could not be used for analysis. The microbiota composition was similar for the control M-SHIME vessel at Week 1 and Week 2 (Figures 2 and 3), allowing for the use of Week 2 data as a comparator in place of Week 1 data. ${ }^{2}$ Values are in $\mathrm{pg} / \mathrm{mL} .{ }^{3}$ Values are in ng/mL. M-SHIME ${ }^{\circledR}=$ mucosal simulator of the human intestinal microbial ecosystem.

The effects of the M-SHIME ${ }^{\circledR}$ suspensions on the NFKB activity of PMA-activated THP1 cells after 6 h LPS stimulation are shown in Figure 6. When pretreated with clindamycin, M-SHIME ${ }^{\circledR}$ suspensions (M-SHIME ${ }^{\circledR}$ control or MegaDuo ${ }^{\mathrm{TM}}$ ) collected after 1 week of MegaDuo $^{\mathrm{TM}}$ dosing induced a slight increase in THP1 NFKB activity, and those collected after 2 weeks of dosing induced a slight decrease in activity compared to the LPS+ control (Figure 6a).

Suspensions not pretreated with clindamycin and collected after Week 1 and 2 of MegaDuo ${ }^{\mathrm{TM}}$ dosing, and M-SHIME ${ }^{\circledR}$ control suspensions collected after Week 2 induced a slight increase in NFKB activity compared to the LPS+ control (Figure $6 \mathrm{~b}$ ). Note that the Week $1 \mathrm{M}-\mathrm{SHIME}{ }^{\circledR}$ control suspensions were suspected to have been contaminated during sample processing (i.e., after sample collection) and were therefore removed from analysis. The microbiota composition was similar for the control M-SHIME vessel at Week 1 and Week 2 (Figures 2 and 3, TR1 vs. TR2), allowing for the use of Week 2 data as a comparator in place of Week 1 data.

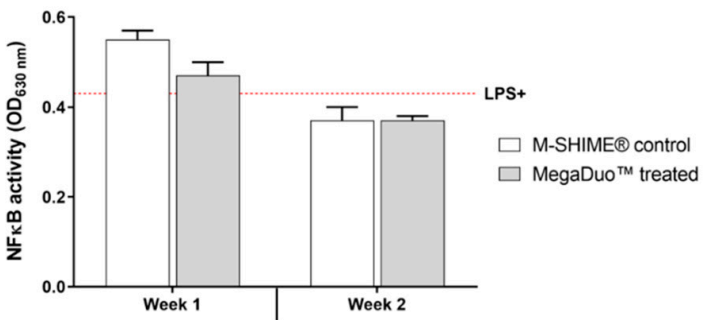

(a)

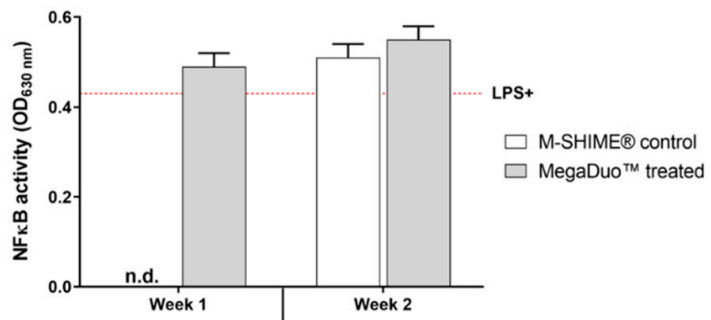

(b)

Figure 6. Effect of M-SHIME ${ }^{\circledR}$ suspensions on NFKB activity of PMA-treated THP1 cells after LPS stimulation in the Caco-2/THP1-Blue ${ }^{\mathrm{TM}}$ co-culture model (a) with or (b) without pre-treatment with clindamycin. Red dashed line = LPS (no M-SHIME ${ }^{\circledR}$ supernatants). Error bars represent standard error of the mean. LPS = lipopolysaccharide; $\mathrm{M}-\mathrm{SHIME}{ }^{\circledR}=$ mucosal simulator of the human intestinal microbial ecosystem.

There were no significant differences between the control and MegaDuo ${ }^{\mathrm{TM}}$ treated M-SHIME ${ }^{\circledR}$ suspensions in terms of NFKB activity, indicating that any changes observed were related to metabolites present in both the control and treated M-SHIME ${ }^{\circledR}$ suspensions and were independent of the MegaDuo ${ }^{\mathrm{TM}}$ treatment.

\section{Discussion}

We observed that a stable microbial community was established prior to clindamycin exposure and that clindamycin treatment induced changes to the microbial community that would be considered 
dysbiosis, including an increase in Enterobacteriaceae. MegaDuo ${ }^{\mathrm{TM}}$ treatment seemed to have a beneficial effect on recovery of the microbial community after antibiotic exposure, as evidenced by a more rapid decrease in Enterobacteriaceae and an increase in SCFA-producing Bacteroidetes and Firmicutes. MegaDuo $^{\mathrm{TM}}$ treatment had beneficial effects on the microbial community under healthy conditions, which included increases in the abundance of Bacteroidetes and Firmicutes. There was an increase in the abundance of the Bacillaceae family in the lumen when MegaDuo ${ }^{\mathrm{TM}}$ treatment was given under conditions of dysbiosis and an increase in both the lumen and mucosal compartments after MegaDuo ${ }^{\mathrm{TM}}$ treatment under healthy conditions (vs. pre-treatment abundance). These results suggest that bacteria in the probiotic formulation may have grafted in the proximal colon chamber.

MegaDuo $^{\mathrm{TM}}$ dosed M-SHIME ${ }^{\circledR}$ suspensions provided an improvement in gut barrier integrity and reduced TNF $\alpha$, MCP1, and IL-6 compared with control M-SHIME ${ }^{\circledR}$ suspensions when under conditions of antibiotic-induced gut microbiome dysbiosis. We speculate that the reductions in cytokines may be attributed to SCFA production by Firmicutes, which recovered to a greater extent in the lumen after MegaDuo ${ }^{\mathrm{TM}}$ treatment compared with no treatment. No significant differences were observed in NFKB activity between MegaDuo ${ }^{\mathrm{TM}}$ treated and M-SHIME ${ }^{\circledR}$ control suspensions, and no significant changes in IL-10 levels were observed regardless of the experimental conditions. In healthy gut conditions (without antibiotic treatment), the pro-inflammatory chemokines TNF $\alpha$, IL- 8 , and MCP1 were significantly decreased with MegaDuo $^{\mathrm{TM}}$ treatment (versus M-SHIME ${ }^{\circledR}$ control); no other significant differences were observed. We speculate that the decrease in TNF $\alpha$, IL- 8 , and MCP1 were the result of positive effects of the MegaDuo ${ }^{\mathrm{TM}}$ probiotics on the abundance of Firmicutes and Bacteroidetes, as both are known to produce SCFAs [43,44], which have an anti-inflammatory effect [12].

It seems necessary for dysbiosis of the normal gut microbiome to occur for a probiotic to exert observable benefits in a relatively short ( 2 week) treatment period. One of the most common side-effects of antibiotic treatment is antibiotic associated diarrhea, which is thought to be caused by a drastic change in the intestinal microbiome (i.e., dysbiosis) [45]. In a clinical trial, B. subtilis was shown to reduce the incidence and severity of antibiotic associated diarrhea in patients receiving antibiotic treatment [46]. However, there is a lack of knowledge regarding the mechanism(s) responsible for this improvement. The results from the present study help bridge this gap by providing mechanistic insight. We observed a reduction in TNF $\alpha$ and MCP1 along with protection from LPS-induced barrier damage in an in vitro model of antibiotic-induced gut microbiome dysbiosis after treatment with the probiotic MegaDuo ${ }^{\mathrm{TM}}$. These results suggest that treatment with the probiotic MegaDuo ${ }^{\mathrm{TM}}$ may reduce cytokine and chemokine production and provide protection from barrier damage under conditions of antibiotic-induced dysbiosis. While these results are of interest, additional studies using fecal microbiota from multiple healthy donors are necessary to further elucidate and validate these findings.

Gut barrier integrity is critically important for healthy intestinal function. One protective mechanism of a healthy gut barrier is the separation of the luminal contents from the underlying immune cells by mucus and a single layer of epithelial cells. The integrity of this membrane is crucial to prevent infection as well as unnecessary, uncontrolled intestinal inflammation. Many gut-associated disorders are characterized by inflammation and a compromised gut barrier [47]. Given the reduction in $\mathrm{TNF} \alpha$ and improvement in gut barrier function after probiotic treatment observed in our study, we think that studies of the effects of $\mathrm{MegaDuo}^{\mathrm{TM}}$ in gut-associated disorders are warranted. A previous clinical trial reported that B. coagulans GBI-30, 6086 probiotic treatment decreased abdominal pain and bloating in IBD patients [48], providing some support for the beneficial effects of probiotic treatment in gut-associated disorders.

Patients having other diseases associated with compromised gut barrier integrity have experienced improvement with Bacillus spp. probiotic use, again with little understanding of the mechanisms behind this improvement. Probiotic treatment with B. coagulans GBI-30, 6086 resulted in quality of life improvement and reduction of gastrointestinal symptoms in a clinical trial of patients with post-prandial gas-related symptoms [49]. Another clinical trial reported a reduction of daily bowel movements in patients with IBD who were treated with B. coagulans GBI-30, 6086 [50]. Our study provides some 
much-needed insight into possible mechanisms behind the clinical improvements observed with Bacillus spp. probiotic treatment and provides support for testing the effects of MegaDuo ${ }^{\mathrm{TM}}$, a unique combination of B. coagulans SC208 and B. subtilis HU58, in patients with gut-associated disorders, particularly in cases where the normal gut microbiome is no longer intact.

Limitations of our study include the fact that the results were obtained using the gut microbiome of a single healthy donor and that the only stressor studied was antibiotic-induced dysbiosis. While M-SHIME ${ }^{\circledR}$ experiments with the aim of generalizing the effect of a treatment in a given population frequently include multiple donors to account for the potential effects of interindividual variability, the present study aimed to evaluate the impact of a test product when comparing the same donor under normal conditions and conditions of antibiotic-induced dysbiosis. Therefore, we think that the data presented are self-standing, representing an exploratory data set that can be used to guide larger, confirmatory studies. Additionally, M-SHIME ${ }^{\circledR}$ control suspensions from Week 1 were suspected to have been contaminated during sample processing (i.e., after sample collection). Because of this, we used data from Week 2 as a comparator for Week 1. While we acknowledge that this could have influenced the interpretation of the results, we expect this to be minimal, as the microbiota composition for the M-SHIME ${ }^{\circledR}$ control was similar between Weeks 1 and 2 (Figures 2 and 3, TR1 vs. TR2).

Overall, this study showed that under conditions of antibiotic-induced dysbiosis in the M-SHIME ${ }^{\circledR}$ system, suspensions from MegaDuo ${ }^{\mathrm{TM}}$ treated colonic chambers could significantly reduce gut membrane barrier damage and significantly decrease TNF $\alpha, \mathrm{MCP} 1$, and IL-6 compared with the M-SHIME ${ }^{\circledR}$ control (no probiotic treatment) in an in vitro co-culture system representing the gut mucosal barrier. The greatest effect was observed when the M-SHIME ${ }^{\circledR}$ suspensions were collected after 2 weeks of treatment with MegaDuo ${ }^{\circledR}$.

Supplementary Materials: The following are available online at http://www.mdpi.com/2076-2607/8/7/1028/s1, Table S1: Reciprocal Simpson Diversity Index in the lumen and mucus of the proximal colon (M-SHIMEß) treated with MegaDuo ${ }^{\mathrm{TM}}$, Figure S1: Barrier integrity of Caco-2 cells (controls), Figure S2: Effect of controls on cytokine and chemokine levels in the Caco-2/THP1-Blue ${ }^{\mathrm{TM}}$ co-culture model, Figure S3: Effect of controls on NFkB activity of PMA-treated THP1 cells after LPS stimulation in the Caco-2/THP1-Blue ${ }^{\mathrm{TM}}$ co-culture model.

Author Contributions: Conceptualization, methodology, and writing-review and editing; M.M., P.V.d.A., T.B., K.K., A.Y., D.M., and A.D.; data curation; M.M. and P.V.d.A.; formal analysis; M.M., P.V. and S.S.B.; writing-original draft preparation and visualization, S.S.B. All authors have read and agreed to the published version of the manuscript.

Funding: This research was funded by Synergia Life Sciences. Article processing charges were funded by Microbiome Labs.

Conflicts of Interest: T.B., K.K., and A.Y. are employees of Microbiome Labs. D.M. and A.D. are employees of Synergia Life Sciences, which produces the MegaDuo ${ }^{\mathrm{TM}}$ spores. S.S.B. received payment from Microbiome Labs. The funders were involved in the design of the study, in the writing of the manuscript, and in the decision to publish the results.

\section{References}

1. Sender, R.; Fuchs, S.; Milo, R. Revised estimates for the number of human and bacteria cells in the body. PLoS Biol. 2016, 14, e1002533. [CrossRef]

2. Jandhyala, S.M.; Talukdar, R.; Subramanyam, C.; Vuyyuru, H.; Sasikala, M.; Reddy, D.N. Role of the normal gut microbiota. World J. Gastroenterol. 2015, 21, 8787-8803. [CrossRef]

3. Human Microbiome Project Consortium. Structure, function and diversity of the healthy human microbiome. Nature 2012, 486, 207-214. [CrossRef]

4. Virgin, H.W.; Todd, J.A. Metagenomics and personalized medicine. Cell 2011, 147, 44-56. [CrossRef]

5. Priyadarshini, M.; Kotlo, K.U.; Dudeja, P.K.; Layden, B.T. Role of short chain fatty acid receptors in intestinal physiology and pathophysiology. Compr. Physiol. 2018, 8, 1091-1115. [PubMed]

6. Peng, L.; He, Z.; Chen, W.; Holzman, I.R.; Lin, J. Effects of butyrate on intestinal barrier function in a Caco-2 cell monolayer model of intestinal barrier. Pediatr. Res. 2007, 61, 37-41. [CrossRef] [PubMed] 
7. Francescone, R.; Hou, V.; Grivennikov, S.I. Cytokines, IBD, and colitis-associated cancer. Inflamm. Bowel. Dis. 2015, 21, 409-418. [CrossRef] [PubMed]

8. Neumann, C.; Scheffold, A.; Rutz, S. Functions and regulation of T cell-derived interleukin-10. Semin. Immunol. 2019, 44, 101344. [CrossRef] [PubMed]

9. Satsu, H.; Ishimoto, Y.; Nakano, T.; Mochizuki, T.; Iwanaga, T.; Shimizu, M. Induction by activated macrophage-like THP-1 cells of apoptotic and necrotic cell death in intestinal epithelial Caco-2 monolayers via tumor necrosis factor-alpha. Exp. Cell Res. 2006, 312, 3909-3919. [CrossRef] [PubMed]

10. Foey, A.D. Butyrate regulation of distinct macrophage subsets: Opposing effects on M1 and M2 macrophages. Int. J. Probiotics Prebiotics 2001, 6, 147-158.

11. Larraufie, P.; Doré, J.; Lapaque, N.; Blottière, H.M. TLR ligands and butyrate increase Pyy expression through two distinct but inter-regulated pathways. Cell Microbiol. 2017, 19.

12. Vinolo, M.A.R.; Rodrigues, H.G.; Nachbar, R.T.; Curi, R. Regulation of inflammation by short chain fatty acids. Nutrients 2011, 3, 858-876. [CrossRef] [PubMed]

13. Sidhu, M.; van der Poorten, D. The gut microbiome. Aust. Fam. Physician 2017, 46, 206-211. [PubMed]

14. Dudek-Wicher, R.K.; Junka, A.; Bartoszewicz, M. The influence of antibiotics and dietary components on gut microbiota. Prz. Gastroenterol. 2018, 13, 85-92. [CrossRef]

15. Knoop, K.A.; McDonald, K.G.; Kulkarni, D.H.; Newberry, R.D. Antibiotics promote inflammation through the translocation of native commensal colonic bacteria. Gut 2016, 65, 1100-1109. [CrossRef]

16. Tulstrup, M.V.; Christensen, E.G.; Carvalho, V.; Linninge, C.; Ahrné, S.; Højberg, O.; Licht, T.R.; Bahl, M.I. Antibiotic treatment affects intestinal permeability and gut microbial composition in Wistar rats dependent on antibiotic class. PLoS ONE 2015, 10, e0144854. [CrossRef]

17. Guidelines for the evaluation of probiotics in food. Available online: https://www.who.int/foodsafety/fs_ management/en/probiotic_guidelines.pdf (accessed on 1 June 2020).

18. Pyne, D.B.; West, N.P.; Cox, A.J.; Cripps, A.W. Probiotics supplementation for athletes-clinical and physiological effects. Eur. J. Sport Sci. 2015, 15, 63-72. [CrossRef]

19. Cutting, S.M. Bacillus probiotics. Food Microbiol. 2011, 28, 214-220. [CrossRef]

20. Eishaghabee, F.M.F.; Rokana, N.; Gulhane, R.D.; Sharma, C.; Panwar, H. Bacillus as potential probiotics: Status, concerns, and future perspectives. Front. Microbiol. 2017, 8, 1490. [CrossRef]

21. Kristjansson, J.K. Thermiphilic Bacteria; CRC Press: Boca Raton, FL, USA, 1991; pp. $26-29$.

22. Konuray, G.; Erginkaya, Z. Potential use of Bacillus coagulans in the food industry. Foods 2018, 7, 92. [CrossRef]

23. Hyronimus, B.; Le Marrec, C.; Sassi, A.H.; Deschamps, A. Acid and bile tolerance of spore-forming lactic acid bacteria. Int. J. Food Microbiol. 2000, 61, 193-197. [CrossRef]

24. EFSA Scientific Opinion on the maintenance of the list of QPS biological agents intentionally added to food and feed (2013 update). EFSA J. 2013, 11, 3449.

25. Hong, H.A.; Khaneja, R.; Tam, N.M.K.; Cazzato, A.; Tan, S.; Urdaci, M.; Brisson, A.; Gasbarrini, A.; Barnes, I.; Cutting, S.M. Bacillus subtilis isolated from the human gastrointestinal tract. Res. Microbiol. 2009, 160, 134-143. [CrossRef]

26. Hong, H.A.; To, E.; Baccigalupi, L.; Ricca, E.; Cutting, S.M. Defining the natural habitat of Bacillus spore formers. Res. Microbiol. 2009, 160, 375-379. [CrossRef] [PubMed]

27. Tam, N.K.; Uyen, N.Q.; Hong, H.A.; Duc le, H.; Hoa, T.T.; Serra, C.R.; Henriques, A.O.; Cutting, S.M. The intestinal life cycle of Bacillus subtilis and close relatives. J. Bacteriol. 2006, 188, 2692-2700. [CrossRef] [PubMed]

28. McFarlin, B.K.; Henning, A.L.; Bowman, E.M.; Gary, M.A.; Carbajal, K.M. Oral spore-based probiotic supplementation was associated with reduced incidence of post-prandial dietary endotoxin, triglycerides, and disease risk biomarkers. World J. Gastrointest. Pathophysiol. 2017, 8, 117-126. [CrossRef] [PubMed]

29. Van den Abbeele, P.; Roos, S.; Eeckhaut, V.; MacKenzie, D.A.; Derde, M.; Verstraete, W.; Marzorati, M.; Possemiers, S.; Vanhoecke, B.; Van Immerseel, F.; et al. Incorporating a mucosal environment in a dynamic gut model results in a more representative colonization by lactobacilli. Microb. Biotechnol. 2012, 5, 106-115. [CrossRef]

30. Molly, K.; Vande Woestyne, M.; Verstraete, W. Development of a 5-step multi-chamber reactor as a simulation of the human intestinal microbial ecosystem. Appl. Microbiol. Biotechnol. 1993, 39, 254-258. [CrossRef] 
31. Van den Abbeele, P.; Belzer, C.; Goossens, M.; Kleerebezem, M.; De Vos, W.M.; Thas, O.; De Weirdt, T.; Kerckhof, F.-M.; Van de Wiele, T. Butyrate-producing Clostridium cluster XIVa species specifically colonize mucins in an in vitro gut model. ISME J. 2013, 7, 949-961. [CrossRef]

32. Possemiers, S.; Verthé, K.; Uyttendaele, S.; Verstraete, W. PCR-DDGE-based quantification of stability of the microbial community in a simulator of the human intestinal microbial ecosystem. FEMS Micrbiol. Ecol. 2004, 49, 495-507. [CrossRef]

33. Vilchez-Vargas, R.; Geffers, R.; Suarez-Diez, M.; Conte, I.; Waliczek, A.; Kaser, V.S.; Kralova, M.; Junca, H.; Pieper, D.H. Analysis of the microbial gene landscape and transcriptome for aromatic pollutants and alkane degradation using a novel internally calibrated microarray system. Environ. Microbiol. 2013, 15, 1016-1039. [CrossRef] [PubMed]

34. Guo, X.; Xia, X.; Tang, R.; Zhou, J.; Zhao, H.; Wang, K. Development of a real-time PCR method for Firmicutes and Bacteroidetes in faeces and its application to quantify intestinal population of obese and lean pigs. Lett. Appl. Microbiol. 2008, 47, 367-373. [CrossRef]

35. Rinttilä, T.; Kassinen, A.; Malinen, E.; Krogius, L.; Palva, A. Development of an extensive set of $16 \mathrm{~S}$ rDNA-targeted primers for quantification of pathogenic and indigenous bacteria in faecal samples by real-time PCR. J. Appl. Microbiol. 2004, 97, 1166-1177. [CrossRef]

36. Furet, J.P.; Firmesse, O.; Guormelon, M.; Bridonneau, C.; Tap, J.; Mondot, S.; Doré, J.; Corthier, G. Comparative assessment of human and farm animal faecal microbiota using real-time quantitative PCR. FEMS Microbiol. Ecol. 2009, 68, 351-362. [CrossRef]

37. Nakano, S.; Kobayashi, T.; Funabiki, K.; Matsumura, A.; Nagao, Y.; Yamada, T. Development of a PCR assay for detection of Enterobacteriaceae in foods. J. Food Prot. 2003, 66, 1798-1804. [CrossRef] [PubMed]

38. Klindworth, A.; Pruesse, E.; Schweer, T.; Peplies, J.; Quast, C.; Horn, M.; Glöckner, F.O. Evaluation of general $16 \mathrm{~S}$ ribosomal RNA gene PCR primers for classical and next-generation sequencing-based diversity studies. Nucleic Acids Res. 2013, 41, e1. [CrossRef]

39. Kozich, J.J.; Wescott, S.L.; Baxter, N.T.; Highlander, S.K.; Schloss, P.D. Development of a dual-index sequencing strategy and curation pipeline for analyzing amplicon sequence data on the MiSeq Illumina sequencing platform. Appl. Environ. Microbiol. 2013, 79, 5112-5120. [CrossRef] [PubMed]

40. Schloss, P.D.; Wescott, S.L. Assessing and improving methods used in operational taxonomic unit-based approaches for $16 \mathrm{~S}$ rRNA gene sequence analysis. Appl. Environ. Microbiol. 2011, 77, 3219-3226. [CrossRef] [PubMed]

41. Wang, Q.; Garrity, G.M.; Tiedge, J.M.; Cole, J.R. Naive Bayesian classifier for rapid assignment of rRNA sequences into the new bacterial taxonomy. Appl. Environ. Microbiol. 2007, 73, 5261-5267. [CrossRef] [PubMed]

42. Daguet, D.; Pinheiro, I.; Verheist, A.; Possemiers, S.; Marzorati, M. Arabinogalactan and fructooligosaccharides improve the gut barrier function in distinct areas of the colon in the Simulator of the Human Intestinal Microbial Ecosystem. J. Funct. Foods 2016, 20, 369-379. [CrossRef]

43. Louis, P.; Flint, H.J. Diversity, metabolism and microbial ecology of butyrate-producing bacteria from the human large intestine. FEMS Microbiol. Lett. 2009, 294, 1-8. [CrossRef] [PubMed]

44. Louis, P.; Flint, H.J. Formation of propionate and butyrate by the human colonic microbiota. Environ. Microbiol. 2017, 19, 29-41. [CrossRef] [PubMed]

45. Suva, M.A.; Sureja, V.P.; Kheni, D.B. Novel insight on probiotic Bacillus subtilis: Mechanism of action and clinical applications. J. Curr. Res. Sci. Med. 2016, 2, 65-72. [CrossRef]

46. Horosheva, T.V.; Vodyanoy, V.; Sorokulova, I. Efficacy of Bacillus probiotics in prevention of antibiotic-associated diarrhea: A randomized, double-blind, placebo-controlled clinical trial. JMM Case Rep. 2014, 1, 1-6. [CrossRef]

47. Pastorelli, L.; De Salvo, C.; Mercado, J.R.; Vecchi, M.; Pizarro, T.T. Central role of the gut epithelial barrier in the pathogenesis of chronic intestinal inflammation: Lessons learned from animal models and human genetics. Front. Immunol. 2013, 4, 280. [CrossRef]

48. Hun, L. Bacillus coagulans significantly improved abdominal pain and bloating in patients with IBS. Postrad. Med. 2009, 121, 119-124. [CrossRef] 
49. Kalman, D.S.; Schwartz, H.I.; Alvarez, P.; Feldman, S.; Pezzullo, J.C.; Krieger, D.R. A prospective, randomized, double-blind, placebo-controlled parallel-group dual site trial to evaluate the effects of a Bacillus coagulans-based product on functional intestinal gas symptoms. BMC Gastroenterol. 2009, 9, 85. [CrossRef]

50. Dolin, B.J. Effects of a proprietary Bacillus coagulans preparation on symptoms of diarrhea-predominant irritable bowel syndrome. Methods Find. Exp. Clin. Pharm. 2009, 31, 655-659. [CrossRef] 